\title{
Effektiver Schutz bei subklinischer Depression
}

Fragestellung: Kann ein therapeutengeleitetes Online-Training bei Patienten mit subklinischer Depression die Entwicklung der Vollsymptomatik einer Major Depression (MD) verhindern?

Hintergrund: Schätzungen zufolge können aktuell zur Verfügung stehende Depressionstherapien die MD-bedingte Krankheitslast nur um ein Drittel reduzieren, umso wichtiger ist es, vorzubeugen. Präventive psychologische Interventionen können bei Risikopersonen den Ausbruch einer depressiven Erkrankung verzögern oder verhindern. Die Wirksamkeit internetbasierter psychotherapeutischer Interventionen zur Reduktion depressiver Symptome ist in Metaanalysen nachgewiesen.

Patienten und Methodik: Die randomisierte kontrollierte Studie untersuchte bei Erwachsenen mit subklinischer Depression ein internetbasiertes therapeutengeleitetes Selbsthilfeprogramm auf Basis der kognitiven Verhaltens- und der Problemlö-

Buntrock C, Ebert DD, Lehr D et al. Effect of a web-based guided self-help intervention for prevention of major depression in adults with subthreshold depression. A randomized clinical trial. JAMA 2016; 315: $1854-63$ setherapie (sechs Lektionen à 30 Minuten). Während die Teilnehmer der Interventionsgruppe ( $\mathrm{n}=202)$ zusätzlich Hausaufgaben und schriftlich individualisiertes Feedback zur Motivations- und Adhärenzsteigerung durch einen Online-Coach erhielten, beschränkte sich das Angebot in der Kontrollgruppe ( $\mathrm{n}=204)$ auf internetbasierte Informationen zur Psychoedukation. Ausschlusskriterien waren kein Internetzugang sowie eine Psychotherapie (geplant, aktuell oder in den letzten sechs Monaten abgeschlossen). Primärer Endpunkt war die Zeit bis zum Auftreten einer depressiven Episode innerhalb eines 12-Monats-Zeitraums.

Ergebnisse: Von den 406 randomisierten Teilnehmern (Durchschnittsalter 45 Jahre, 73,9\% Frauen) beendeten 335 (82\%) die Studie. Die Drop-out-Rate unterschied sich nicht signifikant zwischen den Gruppen. Im Durchschnitt nutzten die Teilnehmer das Programm 5,84 Wochen. 84 Teilnehmer (41 \%) der Kontrollgruppe, aber nur 54 (27\%) der Interventionsgruppe entwickelten innerhalb von zwölf Monaten eine MD. Überlebenszeitanalysen zeigten für die Teilnehmer der Interventionsgruppe im Vergleich zur Kontrollgruppe ein 0,59-faches und damit eindeutig geringeres Risiko, eine MD zu entwickeln (95\%-Konfidenzintervall $[\mathrm{KI}] 0,42-0,82 ; \mathrm{p}=0,002)$. Die number needed to treat, um eine MD zu vermeiden, betrug 5,9 (95\%-KI 3,9-14,6).

Schlussfolgerungen: Eine internetbasierte Selbsthilfeintervention mit minimaler therapeutischer Unterstützung ist bei Erwachsenen mit subklinischer Depression geeignet, die MDHäufigkeit signifikant zu reduzieren.

\section{- Kommentar von Isabella Helmreich, Mainz}

\section{Internetbasierte Depressionsprävention - ein Zukunftskonzept}

Die Studie liefert einen wichtigen Beitrag zur weiteren Erforschung präventiver Möglichkeiten, um die Entwicklung einer MD zu verhindern oder zu verzögern. Sie zeigt, dass eine internetbasierte Intervention einen kostengünstigen und effektiven Präventionsansatz darstellen kann. Hervorzuheben sind die Kürze der Intervention und die günstige number needed to treat von 5,9 (vergleichbar mit Zahlen aus Studien zur Antidepressivatherapie in der Primärversorgung [1]). Insgesamt ist die methodische Qualität der Arbeit mit einem Jadad-Score von 4 als gut einzustufen.

Weiterer Forschungsbedarf besteht hinsichtlich der Generalisierbarkeit der Ergebnisse - etwa aufgrund der Selbstselektion der Stichprobe und der daraus resultierenden Stichprobenzusammensetzung (hauptsächlich Frauen mit hohem Bildungsniveau) - sowie der Notwendigkeit eines OnlineCoaches. Die Intervention kam mit minimaler therapeutischer Unterstützung aus: Die durchschnittliche Zeit, die der Coach für die individualisierten Rückmeldungen aufwand, lag bei etwa drei Stunden pro Teilnehmer. Hier bleibt die spannende Forschungs- und Kosteneffizienzfrage offen, inwieweit sich der therapeutische Kontakt noch weiter einschränken lässt, ohne dadurch die Effektivität der Intervention zu senken. Die meisten Erwachsenen mit beginnenden Depressionssympto- men scheuen sich immer noch davor, frühzeitig Hilfe zu suchen [2]. Internetbasierte Formate, die mehr Anonymität vermitteln und niedrigschwellig sowie flächendeckend angeboten werden können, können somit dazu beitragen, einerseits den Ausbruch einer Erkrankung zu verhindern, andererseits aber auch den Weg zur Inanspruchnahme professioneller Hilfe zu bahnen oder im Sinne eines Stepped-Care-Ansatzes das bestehende Versorgungssystem zu komplementieren.

\footnotetext{
1. Arroll B et al. Cochrane Database of Systematic Reviews 2009; Issue 3. Art. No.: CD007954

2. Mack S et al. Int J Methods Psychiatr Res 2014; 23: 289-303
}

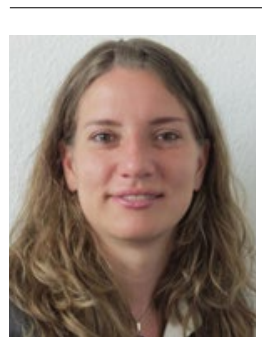

Dr. Dipl.-Psych. Isabella Helmreich, Mainz

Deutsches Resilienz-Zentrum Mainz und Klinik für Psychiatrie und Psychotherapie, Universitätsmedizin Mainz E-Mail: Isabella.Helmreich@unimedizinmainz.de 